\title{
Sequential changes in oligoclonal patterns during the course of multiple sclerosis
}

\author{
EDWARD J THOMPSON, PETER KAUFMANN*, PETER RUDGE \\ From the Institute of Neurology, The National Hospital, Queen Square, London, UK
}

SUMMARY The majority of patients with multiple sclerosis have an abnormality of their spinal fluid immunoglobulins. This alteration is manifested as patterns of diffuse protein bands in the gamma globulin region following electrophoresis. This pattern has been termed one of restricted heterogeneity, or an oligoclonal pattern. We have found changes in the banding pattern of some patients studied longitudinally. Since the significance of these findings may be relevant to the pathogenesis of this notoriously waxing and waning disease, we discuss the importance of methodologies as well as the concept that $\operatorname{IgM}$ and $\operatorname{IgA}$ may be relevant to the interpretation of these observations.

In subacute sclerosing panencephalitis oligoclonal bands present in the gamma globulin region (IgG) on electrophoresis of cerebrospinal fluid (CSF) are absorbed by different measles virus antigens, ${ }^{1}$ and the appearance of bands may change at sequential examinations. ${ }^{23}$ In multiple sclerosis the situation has been the reverse. Thus antigens which can absorb the greater part of the oligoclonal bands have not been found. ${ }^{4}$ Furthermore, the most widely held view (for example Bloom, ${ }^{5}$ Norrby, ${ }^{2}$ Lisak $^{6}$ ) is that once established, the particular pattern of oligoclonal bands found in the CSF of any individual patient, remains the same at subsequent examinations. $^{378}$ The pattern persists, as it were, like a "fingerprint" although there may be changes in density of individual bands. In contrast, our findings show that changes do occur in the oligoclonal patterns on polyacrylamide electrophoresis of unconcentrated CSF from 25 patients with multiple sclerosis studied longitudinally. We suggest that the "fingerprint" concept is spurious.

\section{Material and methods}

We studied 25 patients with multiple sclerosis who had two successive lumbar punctures. They were each examined clinically in detail on several occasions by one of us. Seven Address for reprint requests: Dr EJ Thompson, The Institute of Neurology, Queen Sq, London WC1N 3BG, UK.

Received 30 September 1982. Accepted 20 October 1982

*Present address: 11a Devonshire St London W1N 1FS

This paper was published (J Neurol Neurosurg Psychiatry $1983 ; 46: 115-118)$ with incorrect figures. patients received treatment with antilymphocyte globulin, azathioprine and prednisone as part of a treatment trial. Other patients may or may not have received corticosteroids, vitamins or other unrelated medication at different times during the course of their illness. Polyacrylamide gel electrophoresis was performed on unconcentrated CSF using a modification of the Ornstein and Davis method.9 There was no stacking gel and two gels were run in parallel for each specimen, one for staining with Coomassie Blue and the other with Naphthol Black. Densitometric scanning of the Coomassie Blue stained gels was performed on a Joyce Loebl chromoscan. The results of single studies using this method on a larger group of patients have been reported previously. ${ }^{10}$

\section{Results}

All 25 patients had oligoclonal patterns in CSF electrophoresis at the first examination. Twelve of these showed changes in the pattern at the time of repeat electrophoresis after an interval of between four and 17 months. The changes were confined to that group which had relapsing and remitting disease. None of the six patients with the progressive form of multiple sclerosis showed changes. In eight cases this was due to the appearance of one or more new bands and in four cases due to the loss of bands.

Seven patients received intensive immunosuppressive treatment and three showed changes. Of the other 18 patients nine showed changes. There were also changes in the relative amount of individual bands in many of the patterns. Examples of polyacrylamide gels and densitometric scans are shown in figs $1-4$. 


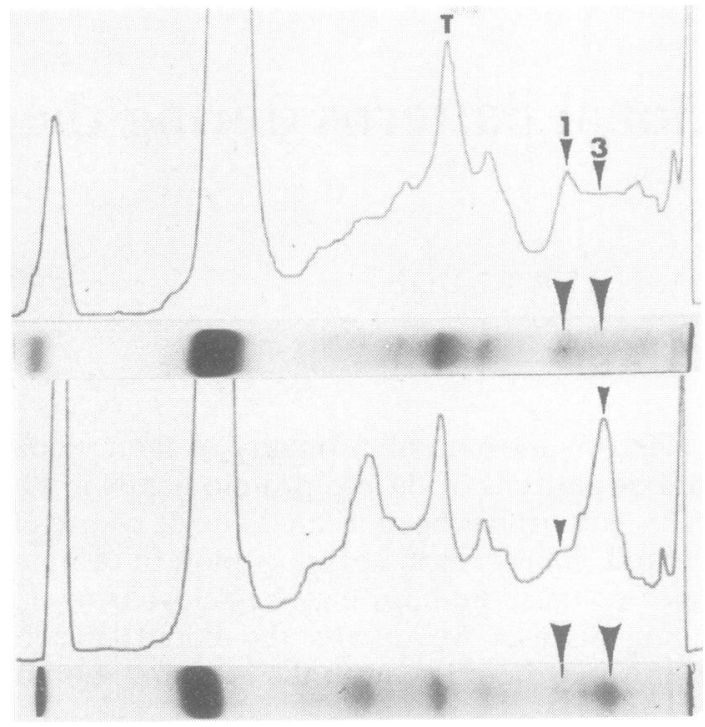

Fig 1 Polyacrylamide gel electrophoresis. $12 \mathrm{~cm}$ gel with cathode to the right. The gamma region is divided into 5 areas, one being the most anodic. Coomassie Blue was used to stain the gels and densitometric scans were performed with a Joyce Loebl chromoscan. The interval between lumbar punctures was 16 months with the gel and scan of the first shown above the second. There is a new gamma 3 band and a diminution of gamma 1 in the second gel and scan. (Arrows indicate bands mentioned on the scan and gel and the respective positions on the other scan and gel.) These changes are relative to transferrin $(T)$ as well as between one gel to the second.

\section{Discussion}

The normal gamma region is shown as a diffuse polyclonal area which on densitometric scanning is a flat trace which varies between specimens in its height above the base line. This background level of gamma globulin is generally higher in multiple sclerosis than in the normal. When an abnormal band is present it is superimposed on the normal area and is visible both to the naked eye and on scanning as a discrete peak with a particular location in the gel ( $R_{f}$ value). Two or more such bands constitute an oligoclonal pattern. The concept of appearance or disappearance of a band depends on the presumption that a band is all new protein superimposed on the existing gamma area rather than that it is just an increase in an existing normal protein of a particular $\mathbf{R}_{\mathrm{f}}$, thus making it stand out. It could be argued that there are never any "new" bands, but just a change in quantity from normal.

In multiple sclerosis, conventional wisdom is that the pattern of bands once established, does not

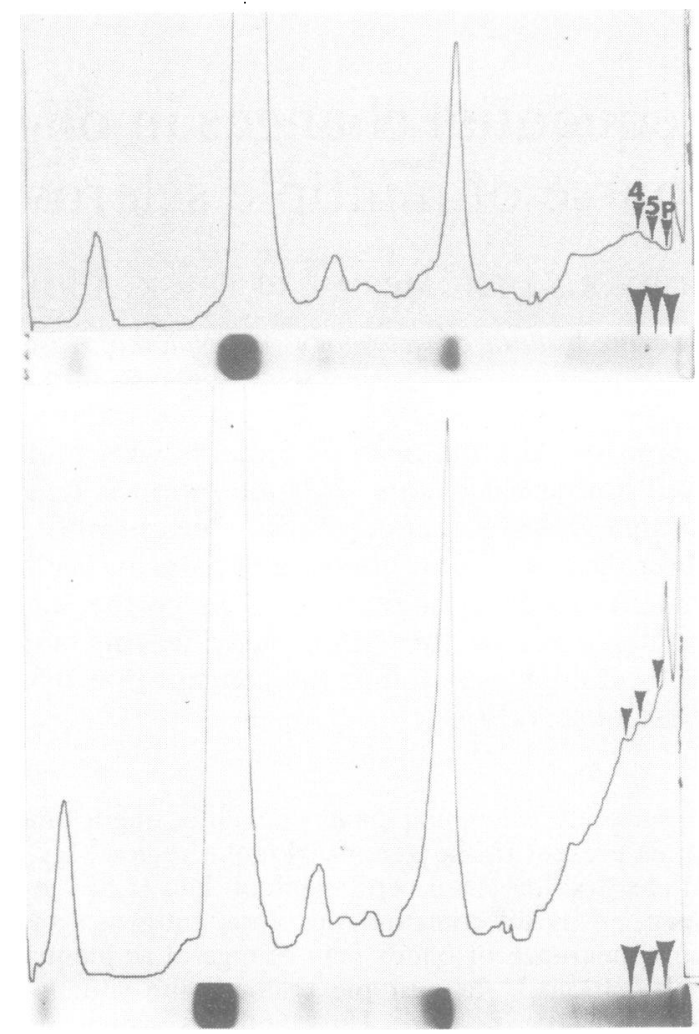

Fig 2 Similar arrangement to fig 1 . Interval 15 months. New gamma 4 and gamma 5 bands as well as new polyclonal post-gamma $(P)$ are present in the second gel and scan.

change at sequential examination during the course of the disease in a particular patient. The number and $R_{f}$ of bands remain the same although the density, or height of peaks, relative to other bands may vary. Concern over the behaviour of such proteins exists because the bands have been shown to be composed mostly of IgG or derivatives, ${ }^{1}$ only a small part of which can be accounted for in terms of activity against known antigens. Small amounts of the IgG have been shown to be specific for measles, herpes simplex and rubella viruses. ${ }^{4}$ This is in contrast to subacute sclerosing panencephalitis where the majority of the oligoclonal IgG has been shown to have measles virus specific activity. Rubella virus panencephalitis, as well as acute mumps and herpes encephalitis are also diseases in which appropriate specificity has been demonstrated. Some discussion of the background to present concepts is therefore important.

Olsson and Link ${ }^{7}$ concluded that the number and 


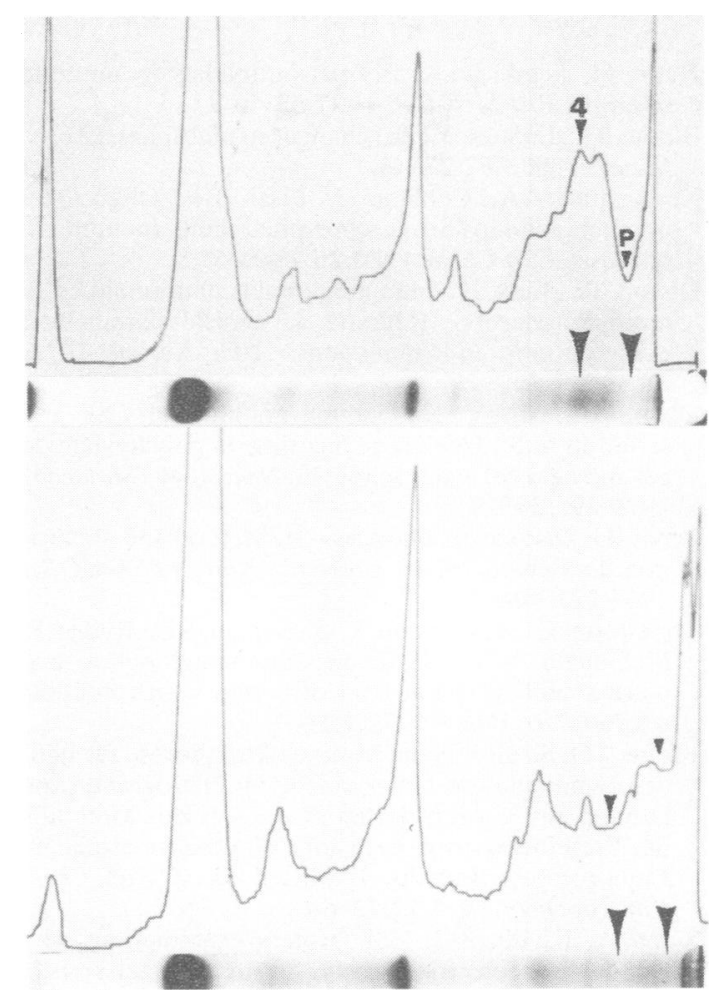

Fig 3 Similar arrangement to fig 1. Interval 15 months. The second gel and scan show the presence of a new post-gamma $(P)$ and the loss of gamma 4.

$R_{\mathrm{f}}$ of the bands demonstrated on agarose electrophoresis of sequential CSF specimens from the same patient did not change. However, this was after they had noted that changes did occur on agar electrophoresis and they dismissed these as due to the methodology. It is to be noted that the agar electrophoresis was performed within 48 hours of receipt of specimens though the studies on agarose were after longer periods of storage which is known to result in the loss of proteins. Furthermore, both methods require concentration of the CSF which also results in some loss of proteins, including $\gamma$-globulin. ${ }^{11}$

Using agarose electrophoresis and concentrated CSF, Vandvik ${ }^{3}$ studied just four patients with "slowly progressive" multiple sclerosis, and simply states that there were no "appreciable" changes in the oligoclonal patterns at sequential examination. Olsson and Nilsson ${ }^{8}$ compared isoelectric focusing (IEF) on polyacrylamide gel with agar electrophoresis, using concentrated CSF and did not find changes. However, also using IEF and concentrated

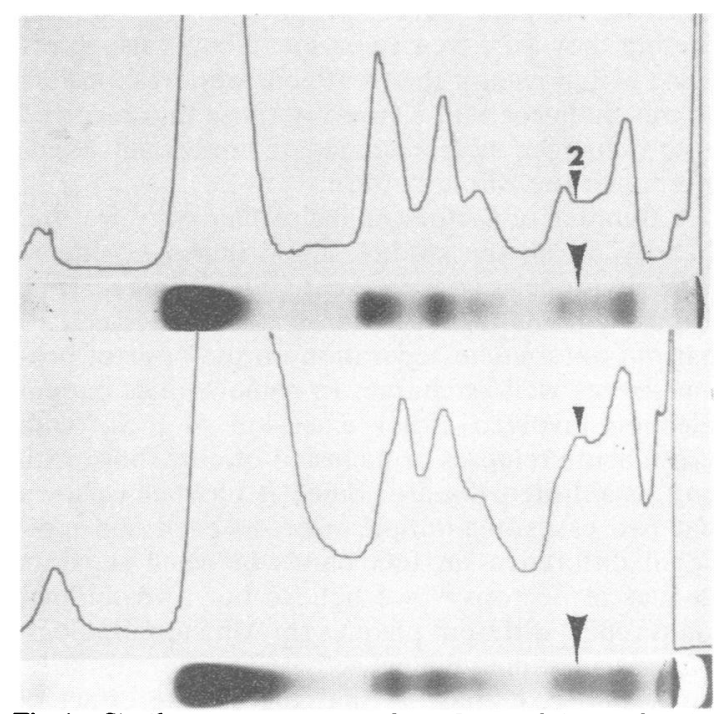

Fig 4 Similar arrangement to fig 1. Interval 4 months. A new gamma 2 band is shown in the second gel and scan.

CSF Sidén and Kjellin ${ }^{12}$ found that abnormal patterns in the gamma region remain unchanged in only 23 out of 46 patients. Presumably the other 23 showed changes. Delmotte and Gonsette ${ }^{13}$ have used IEF to study patients longitudinally and comment that there are no changes in pattern. Mattson et $\mathrm{al}^{14}$ found changes in seven of 10 multiple sclerosis patients using IEF.

Finally, it is worth remembering that patients with previously normal CSF have been shown to develop oligoclonal patterns later in the disease. For instance, in a prospective study of patients with optic neuritis, Sandberg-Wollheim et $a^{15}$ found five who had normal CSF electrophoresis at initial presentation with optic neuritis, but later developed oligoclonal patterns following progression of symptoms typical of multiple sclerosis.

Our findings show that changes do occur in the oligoclonal patterns, and we feel that it is important, in relation to work on the genesis of the bands, that this point be made. The "fingerprint" hypothesis sets limitations. However, what all the work suggests is that there is at least a remarkable tendency towards a stable pattern. Of the many interesting aspects of this, of special relevance is the apparent lack of effect of any treatment. Specifically, Olsson and Nilsson ${ }^{8}$ state that ACTH has no effect and in brief communications Baumhefner et $a l^{16}$ and Brooks et al $^{17}$ also say that high-dose corticosteroid or ACTH therapy has no effect on the incidence of bands. Our results with more potent therapy also support this observation, the alteration of bands 
occurring in the same proportion of patients whether they were treated or not. What is also interesting is that even if the treatment were responsible for the changes that occurred in those that received it, the changes were confined to individual bands and not to the whole pattern.

A number of factors might explain why we find changes. In all the studies supporting the "fingerprint" hypothesis concentrated CSF was used. They also used media (agar or agarose) or methods (IEF) that did not involve separation on the basis of protein size as well as charge. In some studies patient selection involved either exclusion of those with recent acute relapses or inclusion of only those with long established disease. Since it has been shown that two cases of multiple sclerosis each had individual differences in IgG bands between separate plaques at necropsy ${ }^{18}$ we believe our own findings could reflect different plaques showing immunological and sometimes clinical activity at different times. Furthermore, it may be that the sieving effect of polyacrylamide gel is separating the antibody from the larger antigen-antibody complex. ${ }^{19}$ An obvious example of this separation of proteins would be haptoglobin monomers and oligomers which are not discriminated by IEF or agar (agarose) electrophoresis but are widely separated on polyacrylamide.

The occurrence of diffuse bands in the gamma region is due principally to $\mathrm{IgG}$, but oligoclonal IgM and $\operatorname{IgA}$ have also been found recently using IEF (Thompson, in press) as well as with polyacrylamide gel electrophoresis (Johnson and Thompson, data not shown). It may be that the situation parallels that in neurosyphilis, that is IgG represents long term antigenic stimulation whereas IgM represents a more transient response. The obvious question remains: what is the antigenic specificity of these more evanescent patterns? The approach to specific binding of IgG should also be re-evaluated in this light.

This work was supported in part by grants from the Medical Research Council and the Wellcome Trust.

\section{References}

1 Vandvik B, Norrby E, Nordal JH, Degre M. Oligoclonal measles virus-specific IgG antibodies isolated from cerebrospinal fluids, brain extracts, and sera from patients with subacute sclerosing panencephalitis and multiple sclerosis. Scand J Immunol 1976;5:979-92.

${ }^{2}$ Norrby E. In: Karcher D, Lowenthal A, Strosberg AD, eds. Humoral immunity in Neurological Diseases. New York: Plenum Press, 1979:289-97.

${ }^{3}$ Vandvik B. Oligoclonal measles virus-specific-IgG antibodies isolated from sera of patients with subacute sclerosing panencephalitis. Scand J Immunol 1977; 6:913-22.

${ }^{4}$ Haire M. Significance of virus antibodies in multiple sclerosis. Brit Med Bull 1977;33:40-4.

5 Bloom BR. Immunological changes in multiple sclerosis. Nature 1980;287:275-6.

${ }^{6}$ Keshgegian AA, Coblentz J, Lisak RP. Oligoclonal immunoglobulins in cerebrospinal fluid in multiple sclerosis. Clin Chem 1980;26:1340-5.

${ }^{7}$ Olsson JE, Link H. Immunoglobulin abnormalities in multipłe sclerosis. Relation to clinical parameters: exacerbations and remissions. Arch Neurol 1973; 28:392-9.

${ }^{8}$ Olsson JE, Nilsson K. Gamma gobulins of CSF and serum in M.S.: Isoelectric focusing on polyacrylamide gel and agar gel electrophoresis. Neurology (Minneap) 1979;29:1383-91.

9 Davis BJ. Disc electrophoresis-II. Method and application to human serum proteins. Ann NY Acad Sci 1964;121:404-27.

10 Thompson EJ, Kaufmann P, Shortman RC, Rudge P, McDonald WI. Oligoclonal immunoglobulins and plasma cells in spinal fluid of patients with multiple sclerosis. Br Med J 1979;1:16-7.

"Kleine TO, Stroh J. Neue Mikroelektrophorese für nativen Lumballiquor. Unterschiede im Pherogramm von nativen und konzentrierten Proteinen. Zur Methodik der Proteinelektrophorese auf Celluloseacetatfolie im Liquor cerebrospinalis. II Mitteilung. Z Klin Chem Klin Biochem 1974;12:73-80.

12 Sidén A, Kjellin KG. CSF protein examinations with thin-layer isoelectric focusing in multiple sclerosis. $J$ Neurol Sci 1978;39:131-46.

${ }^{13}$ Delmotte P, Gonsette R. Biochemical findings in multiple sclerosis. IV. Isoelectric focusing of the CSF gamma globulins in multiple sclerosis (262 cases) and 흔 other neurological diseases (272 cases). J Neurol $\stackrel{\rightleftharpoons}{\omega}$ 1977;215:27-37.

${ }^{14}$ Mattson DH, Roos RP, Arnason BGW. Comparison of agar gel electrophoresis and isoelectric focusing in multiple sclerosis and subacute sclerosing panencephalitis. Ann Neurol 1981;9:34-41.

15 Sandberg-Wollheim M. Optic neuritis: studies on the cerebrospinal fluid in relation to clinical course in 61 patients. Acta Neurol Scand 1975;52:167-78.

${ }^{16}$ Baumhefner RW, Mendex M, Ma BI, Tourtellotte WW. Modulation of de novo central nervous system (CNS) IgG synthesis with preservation of oligoclonal IgG in multiple sclerosis (MS). Neurology (Minneap) 1979;29:549.

17 Brooks BR, Jubelt B, Cohen S, O'Donnell P, Johnson TR, McKhann GM. Cerebrospinal fluid (CSF) myelin basic protein (MBP) in multiple sclerosis (MS): effect of prolonged high single-dose alternate-day prednisone therapy. Neurology (Minneap) 1979;29:548.

18 Mattson DH, Roos RP, Arnason BGW. Isoelectric focusing of IgG eluted from multiple sclerosis and subacute sclerosing panencephalitis brains. Nature 1980;287:335-7.

${ }^{19}$ Maidment Jr BW, Papsidero LD, Chu TM. Isoelectric focusing-a new approach to the study of immune complexes. J Immunol Meth 1980;35:297-306. 